\title{
Adaptive Inverse Control of a Vibrating Coupled Vessel-Riser System With Input Backlash
}

\author{
Xiuyu $\mathrm{He}^{\circledR}$, Student Member, IEEE, Zhijia Zhao ${ }^{\circledR}$, Member, IEEE, Jinya Su ${ }^{\circledR}$, Member, IEEE, \\ Qinmin Yang ${ }^{\circledR}$, Senior Member, IEEE, and Dachang Zhu
}

\begin{abstract}
This article involves the adaptive inverse control of 2 a coupled vessel-riser system with input backlash and system 3 uncertainties. By introducing an adaptive inverse dynamics of 4 backlash, the backlash control input is divided into a mis5 match error and an expected control command, and then a 6 novel adaptive inverse control strategy is established to elim7 inate vibration, tackle backlash, and compensate for system 8 uncertainties. The bounded stability of the controlled system 9 is analyzed and demonstrated by exploiting the Lyapunov's to criterion. The simulation comparison experiments are finally ${ }_{11}$ presented to verify the feasibility and effectiveness of the control 12 algorithm.
\end{abstract}

Index Terms-Adaptive inverse control, boundary control, 14 flexible risers, input backlash, vibration control.

\section{5 \\ "A} DAPTIVE control as a common method for handling parametric uncertainty, provides techniques and algo18 rithms for parameter estimation and is introduced in many 19 literatures [1]-[6]. In recent decades, significant advance20 ments in adaptive control for the nonlinear systems have 21 been documented. To list some, in [7] and [8], switched 22 nonlinear systems were stabilized by developing an adap23 tive neural tracking control and the semiglobal boundedness 24 was ensured. In [9]-[11], an adaptive finite-time convergence

Manuscript received February 28, 2019; revised June 21, 2019; accepted

September 23, 2019. This work was supported in part by the National Natural Science Foundation of China under Grant 61803109 and Grant 11832009 , in part by the Innovative School Project of Education Department of Guangdong under Grant 2017KQNCX153, Grant 2018KQNCX192, and Grant 2017KZDXM060, in part by the Science and Technology Planning Project of Guangzhou City under Grant 201904010494 and Grant 201904010475, and in part by the Open Research Project of the State Key Laboratory of Industria Control Technology under Grant ICT1900303. This article was recommended by Associate Editor J. Fu. (Corresponding author: Zhijia Zhao.)

$\mathrm{X} . \mathrm{He}$ is with the School of Automation and Electrical Engineering, University of Science and Technology Beijing, Beijing 100083, China, and also with the Institute of Artificial Intelligence, University of Science and Technology Beijing, Beijing 100083, China (e-mail: bhilly@163.com).

Z. Zhao and D. Zhu are with the School of Mechanical and Electrical Engineering, Guangzhou University, Guangzhou 510006, China (e-mail: zhjzhaoscut@163.com; zdc98998@gzhu.edu.cn).

J. Su is with the School of Computer Science and Electronic Engineering, University of Essex, Colchester CO4 3SQ, U.K. (e-mail: j.su@essex.ac.uk).

Q. Yang is with the State Key Laboratory of Industrial Control Technology, Zhejiang University, Hangzhou 310027, China, and also with the College of Control Science and Engineering, Zhejiang University, Hangzhou 310027, China (e-mail: qmyang@zju.edu.cn).

Color versions of one or more of the figures in this article are available online at http://ieeexplore.ieee.org.

Digital Object Identifier 10.1109/TSMC.2019.2944999 control for uncertain nonlinear systems was investigated via 25 parameter estimation. Liu et al. [12], Zhang et al. [13], and 26 Liu et al. [14] explered an adaptive neural control method- 27 ologies for uncertain nonlinear systems subject to constraints. 28 In [15] and [16], an adaptive fuzzy sliding-mode control was 29 designed for nonlinear systems to compensate for unknown 30 upper bounds. However, the aforesaid results were just con- 31 cerned on the adaptive control analysis of ordinary differential 32 equation systems and it cannot be applied in partial differential 33 equation systems.

The flexible marine riser is crucial in the exploitation of 35 ocean petroleum and natural gas resources, and receives more 36 and more attention in recent years [17]. Generally, vibra- 37 tion and deformation appear in flexible risers due to the face 38 of harsh conditions, however, the undesired vibration may 39 shorten service life, lead to fatigue failure, and even cause 40 serious environmental pollution [18]. Hence, how to develop 41 the effective active control strategies [19], [20] for eliminat- 42 ing the riser's vibration has attracted many scholars, and 43 they have presented many control approaches including model 44 reduction method [21]-[23] and boundary control [24]-[27]. 45 Boundary control, the implementation of which is generally 46 considered to be nonintrusive actuation and sensing [28]-[38], ${ }_{47}$ is more realistic and effective for stabilizing flexible riser 48 systems due to the circumvention of control spillover result- 49 ing from the reduced-order model method [39]-[41], and 50 the recent developments have been documented. To men- 51 tion a few examples, in [18], a boundary adaptive control 52 framework was raised for the stabilization of an uncertain 53 flexible riser system. In [42], an anti-disturbance control 54 was put forward to damp the riser's oscillation and real- 55 ize the extrinsic disturbance elimination. In [43], the riser 56 vibration decrease was achieved using the presented bound- 57 ary robust output feedback control, which simultaneously 58 ensured the controlled system state's eonvergence. In [44], 59 three-dimensional (3-D) extensible risers were exponentially 60 stabilized under the designed boundary control scheme. 61 Meanwhile, the well-posedness and stability analysis were 62 also presented. In [45], boundary controllers were proposed to 63 address the large in-plane deflection reduction and the global 64 and exponential stabilization of unshearable and extensible 65 flexible risers subject to sea loads. In [46], 3-D longitudi- 66 nal and transverse vibrations of flexible risers with bending 67 couplings were suppressed via boundary simultaneous con- 68 trollers. However, note that the above-mentioned approaches 69 were confined to suppress vibrations, which are invalid 70 
71 for flexible riser systems with nonsmooth input nonlinear 72 constraints.

73 In recent years, significant attention has foeused on con74 trol of nonlinear systems subject to input nonlinearities, such 75 as backlash, deadzone, saturation, and hysteresis [47]-[53], 76 which are common and tough issues in mechanical 77 connections, piezoelectric translators, and hydraulic servo 78 valves [54]-[56]. Recently, boundary control has achieved 79 rapid development on handling the input constraints in flex80 ible riser systems [57]-[59]. In [60], an input-restricted riser 81 system was significantly stabilized by using anti-saturation 82 vibration control strategies. In [57], backstepping technique 83 was employed to construct an adaptive control for riser 84 systems to resolve the oscillation elimination, input saturation, 85 and output constraint. Further, anti-saturation control strate${ }_{86}$ gies were presented to restrain the oscillation of flexible risers 87 with input constraint by introducing the Nussbaum function 88 in [58]. Note that the chattering phenomenon caused by the 89 discontinuous sign function in [57] was removed. In [59], 90 hybrid input deadzone and saturation constraint issue in the 91 riser system was addressed by exploiting the auxiliary function 92 to propose a boundary control law. However, in the aforemen93 tioned research, the design was confined to eliminate vibration, 94 tackle input saturation, or eliminate mixed input deadzone and 95 saturation in the riser system.

96 However, the effect of the input backlash nonlinearity char97 acteristic was not considered in these mentioned literatures.

98 Backlash, which describes a dynamical input-output relation99 ship, exists in various physical systems and devices, such 100 as electronic relay circuits, mechanical actuators, electro101 magnetism, biology optics, and other areas [50]. The effects 102 of input backlash nonlinearity can seriously deteriorate system 103 performance, give rise to undesirable inaccuracy or oscilla104 tions, and even result in closed-loop instability [50]. In [61], 105 an adaptive control with an adjustable update law were 106 established by discomposing and treating the backlash as ${ }_{107}$ "disturbance-like" items. To the best of our knowledge, despite 108 great advances in boundary control design for flexible riser 109 systems subjected to input nonlinearities have been made, the 110 framework on how to develop an adaptive inverse control for 111 tackling the simultaneous effects of the input backlash non112 linearity and uncertainties in the riser system has not been 113 reported thus far in the literature. It is what to motivate this 114 research and, in this article, we consider and investigate a 115 vessel-riser system depicted in Fig. $1_{1}$ simultaneously affected 116 by input backlash and system uncertainties.

117 The main contributions of this article are summarized as 118 follows: 1) the input backlash is reformulated in a sum of a 119 desired control signal and a mismatch error by introducing 120 an adaptive inverse backlash dynamics, rather than resolving 121 and visualizing the backlash as disturbance-like items and 2) a 122 new adaptive inverse control strategy with online update laws 123 is developed to achieve the vibration attenuation, backlash ${ }_{124}$ elimination, and uncertainties compensation for the coupled 125 vessel-riser system.

126 This article is laid out as follows: a dynamical model 127 of the system and preliminaries are arranged in Section II. ${ }_{128}$ Section III presents the stability analysis and the controller

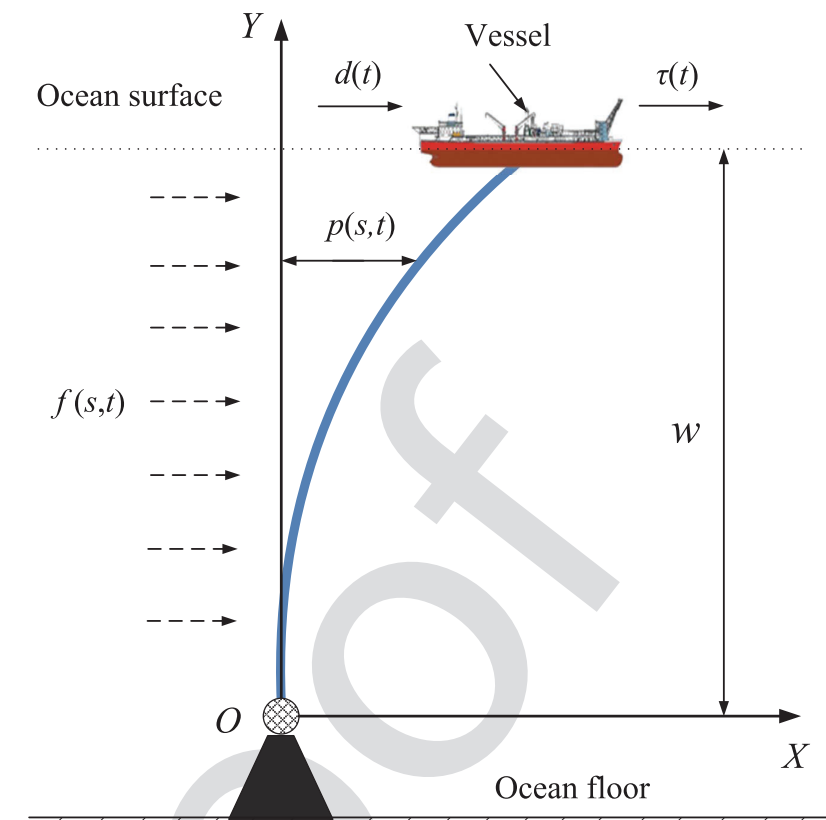

Fig. 1. Vessel-riser system

design. Section IV makes and analyzes numerical simulations. 129 Finally, Section V draws a conclusion.

\section{Problem Statement}

\section{A. System Model}

As displayed in Fig. 1, $p(s, t)$ describes the vibrational ${ }_{133}$ deflection of the riser whose length is $w . d(t)$ denotes the ${ }_{134}$ extraneous disturbance acting on the vessel whose mass and ${ }_{135}$ damping coefficient are $m>0$ and $d_{a}>0 . f(s, t)$ denotes the ${ }_{136}$ distributed disturbance which is acted on the riser, and $\tau(t){ }_{137}$ represents control input which is put on the vessel. In addition, 138 some notations for simplification are presented: $(\dot{*})=\partial(*) / \partial t,{ }_{139}$ $(*)^{\prime}=\partial(*) / \partial s,(\dot{*})^{\prime}=\partial^{2}(*) / \partial s \partial t, \quad(*)^{\prime \prime}=\partial^{2}(*) / \partial s^{2},{ }_{140}$ $(*)^{\prime \prime \prime}=\partial^{2}(*) / \partial s^{3},(*)^{\prime \prime \prime \prime}=\partial^{2}(*) / \partial s^{4}$, and $(\ddot{*})=\partial^{2}(*) / \partial t^{2} . \quad{ }_{141}$

In this article, the goal is to propose an adaptive inverse con- 142 trol for damping the vibration deflection and simultaneously ${ }_{143}$ handling the backlash nonlinearity and system uncertainties. ${ }_{144}$ To realize this objective, we model the dynamics of the 145 considered vessel-riser system as [18]

$$
\begin{aligned}
& \rho \ddot{p}+E I p^{\prime \prime \prime \prime}-T p^{\prime \prime}+c \dot{p}-f=0,0<s<w \\
& p(0, t)=p^{\prime}(0, t)=p^{\prime \prime}(w, t)=0 \\
& m \ddot{p}(w, t)+T p^{\prime}(w, t)-E I p^{\prime \prime \prime}(w, t) \\
& +d_{a} \dot{p}(w, t)=\tau(t)+d(t)
\end{aligned}
$$

where $E I>0, c>0, \rho>0$, and $T>0$ express the bend- ${ }_{151}$ ing stiffness, damping coefficient, mass per unit length, and ${ }_{152}$ tension of the riser, respectively.

\section{B. Input Backlash Analysis}

154

For the convenience of adaptive inverse control design, we ${ }_{155}$ present the expression of the backlash nonlinearity [62] shown 156 


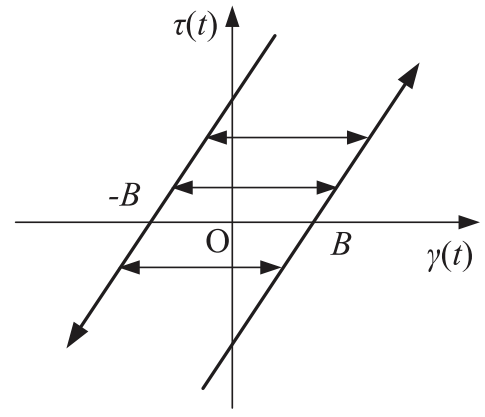

Fig. 2. Backlash nonlinearity.

157 in Fig. 2 as follows:

$$
\begin{aligned}
{ }_{158} \tau(t) & =\mathcal{B}(\gamma) \\
& = \begin{cases}\varrho(\gamma(t)-B), & \text { if } \dot{\gamma}>0 \text { and } \tau(t)=\varrho(\gamma(t)-B) \\
\varrho(\gamma(t)+B), & \text { if } \dot{\gamma}<0 \text { and } \tau(t)=\varrho(\gamma(t)+B) \\
\tau\left(t_{-}\right), & \text {otherwise }\end{cases}
\end{aligned}
$$

160

161 where $\tau(t)$ denotes the control input, $\gamma(t)$ expresses the 162 expected control to be developed, $\varrho$ represents the slope, ${ }_{163} B$ denotes the "crossing," and $\tau\left(t_{-}\right)$shows no change in $\tau(t)$.

\section{${ }_{164}$ C. Preliminaries}

165 We provide the related assumptions, lemmas, and remarks 166 for facilitating the subsequent analysis and design.

${ }_{167}$ For disturbances $d(t)$ and $f(s, t)$ that possess finite energy, ${ }_{168}$ that is, $d(t), f(s, t) \in \mathcal{L}_{\infty}[63]-[65]$, we make an assumption 169 for these disturbances in the following.

170 Assumption 1: We assume that extraneous disturbances $d(t)$ 171 and $f(s, t)$ acting on the vessel and riser are bounded and there ${ }_{172}$ exist $D, F \in \mathbb{R}^{+}$satisfying $|d(t)| \leq D$, and $|f(s, t)| \leq F$, $173 \forall(s, t) \in[0, w] \times[0,+\infty)$.

$174 \quad$ Lemma 1 [66]: Let $\delta_{1}(s, t), \delta_{2}(s, t) \in \mathbb{R}, \phi>0$ with $175(s, t) \in[0, w] \times[0,+\infty)$, then

$$
{ }_{176} \delta_{1} \delta_{2} \leq \frac{1}{\phi} \delta_{1}^{2}+\phi \delta_{2}^{2} .
$$

177 Lemma 2 [67]: Let $\delta(s, t) \in \mathbb{R}$ be under the condition $178 \delta(0, t)=0$, where $(s, t) \in[0, w] \times[0,+\infty)$, then

$$
{ }_{179} \leq w \int_{0}^{w} \delta^{\prime 2} d s .
$$

180 Lemma 3 [68]: The following inequality is provided to 181 derive our main results:

${ }_{182} \quad 0 \leq|\varpi(t)|-\varpi(t) \tanh (\varpi(t)) \leq 0.2785$.

\section{Control Design}

184 System parameters $T, E I, d_{a}$, and $m$, and upper bounds of 185 the disturbance $D$ are utilized in the control design. However, 186 these parameters may be unavailable in real system, thus it ${ }_{187}$ will bring a challenge for the control design and even make 188 control approaches unusable. In this section, we present a 189 new adaptive inverse controller to handle system uncertainties. 190 Moreover, this control scheme can stabilize the vessel-riser system and eliminate the backlash nonlinearity. Subsequently, 191 we analyze the closed-loop system's stability in theory.

\section{A. Boundary Adaptive Inverse Control With Input Backlash}

Invoking [62], the inverse backlash is presented as follows: 194

$$
\gamma^{*}(t)=\mathcal{B I}\left(\tau_{d}(t)\right)=\left\{\begin{array}{cc}
\frac{1}{\varrho} \tau_{d}(t)+B, & \text { if } \dot{\tau_{d}}(t)>0 \\
\frac{1}{\varrho} \tau_{d}(t)-B, & \text { if } \dot{\tau_{d}}(t)<0 \\
\gamma^{*}\left(t_{-}\right), & \text {otherwise }
\end{array}\right.
$$

where $\mathcal{B I}(\cdot)$ represents a backlash inverse function and $\tau_{d}(t){ }_{196}$ denotes the expected control command.

Then, according to the above analysis and [62], we propose 198 an adaptive inverse of backlash as

$$
\begin{aligned}
& \dot{\alpha}(t)=\dot{\tau}_{d}(t)-\frac{\beta}{B_{m}}\left|\dot{\tau}_{d}(t)\right| \alpha(t) \\
& \gamma(t)=\widehat{\mathcal{B I}}\left(\tau_{d}(t)\right)=\frac{1}{\varrho} \tau_{d}(t)+\widehat{\lambda} \beta \alpha(t)
\end{aligned}
$$

where $\beta$ denotes a positive gain parameter, $B_{m}$ denotes a nomi- 202 nal backlash value, $\widehat{\mathcal{B I}}(\cdot)$ denotes an adaptive backlash inverse 203 compensator, and $\widehat{\lambda}$ represents an adaption to adjust $B_{m}$ so 204 as to match the actual backlash spacing $B$ with $B=\lambda B_{m}, 205$ $\lambda \leq \varepsilon, \varepsilon \in \mathbb{R}^{+}$, and $\tilde{\lambda}=\widehat{\lambda}-\lambda$.

Meanwhile, we bring a mismatch error as follows:

$$
\tau(t)=\mathcal{B}\left(\widehat{\mathcal{B I}}\left(\tau_{d}(t)\right)\right)=\tau_{d}(t)+\tau_{e}(t)
$$

where we formulate the mismatch error $\tau_{e}(t)$ as

$$
\tau_{e}(t)=\varrho \tilde{\lambda} B_{m} \operatorname{sgn}\left(\dot{\tau}_{d}(t)\right)=\varrho \tilde{\lambda} \beta \alpha(t) .
$$

Invoking (9)-(12), we then rewrite (3) as

$$
\begin{aligned}
& m \ddot{p}(w, t)+T p^{\prime}(w, t)-E I p^{\prime \prime \prime}(w, t)+d_{a} \dot{p}(w, t)-d(t) \\
& \quad=\tau(t)=\mathcal{B}(\gamma(t))
\end{aligned}
$$

Then, an adaptive inverse control is proposed as

$$
\gamma(t)=\widehat{\mathcal{B I}}\left(\tau_{d}(t)\right)=\frac{1}{\varrho} \tau_{d}(t)+\widehat{\lambda} \beta \alpha(t)
$$

where $\tau_{d}(t)$ is designed as

$$
\begin{aligned}
\tau_{d}(t)= & -\kappa_{1} x(t)-\widehat{E I} p^{\prime \prime \prime}(w, t)+\widehat{T} p^{\prime}(w, t)+\widehat{d}_{a} \dot{p}(w, t) \\
& +\widehat{m}\left(\kappa_{2} \dot{p}^{\prime \prime \prime}(w, t)-\kappa_{3} \dot{p}^{\prime}(w, t)\right)-\tanh (x(t)) \hat{D}
\end{aligned}
$$

where $\kappa_{1}, \kappa_{2}, \kappa_{3}>0, \widehat{D}, \widehat{T}, \widehat{E I}, \widehat{d}_{a}$, and $\widehat{m}$ are the estimated 219 values of $D, T, E I, d_{a}$, and $m$, and we define the auxiliary 220 variable $x(t)$ as

$$
x(t)=\dot{p}(w, t)-\kappa_{2} p^{\prime \prime \prime}(w, t)+\kappa_{3} p^{\prime}(w, t) .
$$

Now, we present the following inverse backlash dynamics: 223

$$
\dot{\alpha}(t)=\dot{\tau}_{d}(t)-\frac{\beta}{B_{m}}\left|\dot{\tau}_{d}(t)\right| \alpha(t)
$$

and the estimation $\widehat{\lambda}$ is obtained from the following:

$$
\dot{\hat{\lambda}}=-\frac{\varrho}{v} \beta x(t) \alpha(t)-\widehat{\lambda} .
$$

At this time, the adaptive laws are presented when system 227 parameters $T, E I, d_{a}$, and $m$, and upper bounds of the ${ }_{228}$ disturbance $D$ are not available

$$
\hat{D}=x(t) \tanh (x(t))-\varsigma_{1} \widehat{D}
$$




$$
\begin{aligned}
& { }_{231} \hat{T}=-x(t) p^{\prime}(w, t)-\varsigma_{2} \widehat{T} \\
& \widehat{E I}=x(t) p^{\prime \prime \prime}(w, t)-\varsigma_{3} \widehat{E I} \\
& \hat{\widehat{d}}_{a}=-x(t) \dot{p}(w, t)-\varsigma_{4} \widehat{d}_{a} \\
& \hat{m}=x(t)\left(\kappa_{3} \dot{p}^{\prime}(w, t)-\kappa_{2} \dot{p}^{\prime \prime \prime}(w, t)\right)-\varsigma_{5} \widehat{m}
\end{aligned}
$$

235 where $\varsigma_{i}>0, i=1 \cdots 5$.

236 The estimation errors are defined as

$$
\begin{aligned}
\widetilde{D} & =\widehat{D}-D, \widetilde{T}=\widehat{T}-T, \\
\widetilde{E} I & =\widehat{E I}-E I, \widetilde{d}_{a}=\widehat{d}_{a}-d_{a}, \widetilde{m}=\widehat{m}-m .
\end{aligned}
$$

Remark 1: Note that available boundary signals $p^{\prime \prime \prime}(w, t)$, $240 p^{\prime}(w, t), p(w, t), \dot{p}^{\prime \prime \prime}(w, t), \dot{p}^{\prime}(w, t)$, and $\dot{p}(w, t)$ consist of the 241 proposed control law (15), where $p^{\prime \prime \prime}(w, t)$ is measured by 242 shear force sensors, $p^{\prime}(w, t)$ is measured by inclinometers, and ${ }_{243} p(w, t)$ is measured by laser displacement sensors. Moreover, 244 we can use the backward difference algorithm to achieve the 245 one-order time derivative of some measurable signals $\dot{p}^{\prime \prime \prime}(w, t)$, $246 \dot{p}^{\prime}(w, t)$, and $\dot{p}(w, t)$ in the designed controller.

247 Now, we will present the stability analysis for deriving our 248 main results.

\section{B. Stability Proof}

252 where

253 $\Theta_{1}(t)=\frac{\xi}{2} E I \int_{0}^{w} p^{\prime \prime 2} d s+\frac{\xi}{2} \rho \int_{0}^{w} \dot{p}^{2} d s+\frac{\xi}{2} T \int_{0}^{w} p^{\prime 2} d s$

254

255

256

257

258

259 with $\xi, \sigma, \psi>0$ being constants.

260

261

262

263 where $\iota_{1}, \iota_{2}>0$

264

266 where $\vartheta=(\psi \rho w / \min (\xi \rho, \xi T, \xi E I))$.

267 We select $\xi$ and $\psi$ appropriately to satisfy the following:

$$
\xi>\frac{\psi \rho w}{\min (\rho, T, E I)} .
$$

269 270 and adding (26) gives

271

$$
0 \leq(1-\vartheta) \Theta_{1}(t) \leq \Theta_{1}(t)+\Theta_{4}(t) \leq(1+\vartheta) \Theta_{1}(t) .
$$

Invoking (25) and (33) yields

$$
\begin{aligned}
0 & \leq \iota_{1}\left[\Theta_{1}(t)+\Theta_{2}(t)+\Theta_{3}(t)\right] \leq \Theta(t) \\
& \leq \iota_{2}\left[\Theta_{1}(t)+\Theta_{2}(t)+\Theta_{3}(t)\right]
\end{aligned}
$$

where $\iota_{1}=\min (1-\vartheta, 1)>0$ and $\iota_{2}=\max (1+\vartheta, 1)>1$

Lemma 5: The time derivative of (25) is upper bounded as 276

$$
\dot{\Theta}(t) \leq-\iota \Theta(t)+\chi
$$

where $\iota, \chi>0$.

Proof: We differentiate (25) to derive

$$
\dot{\Theta}(t)=\dot{\Theta}_{1}(t)+\dot{\Theta}_{2}(t)+\dot{\Theta}_{3}(t)+\dot{\Theta}_{4}(t) .
$$

Invoking (1) and applying Lemma $1, \dot{\Theta}_{1}(t)$ is obtained as 281

$$
\begin{aligned}
\dot{\Theta}_{1}(t) \leq & \frac{\xi E I}{2 \kappa_{2}} x^{2}(t)-\frac{\xi E I \kappa_{2}}{2} p^{\prime \prime \prime 2}(w, t)-\frac{\xi E I}{2 \kappa_{2}} \dot{p}^{2}(w, t) \\
& -\frac{\xi E I \kappa_{3}^{2}}{2 \kappa_{2}} p^{\prime 2}(w, t)+\left(\xi T-\frac{\xi E I \kappa_{3}}{\kappa_{2}}\right) p^{\prime}(w, t) \dot{p}(w, t) \\
& +\xi E I \kappa_{3} p^{\prime \prime \prime}(w, t) p^{\prime}(w, t)-\left(c-v_{1}\right) \xi \int_{0}^{w} \dot{p}^{2} d s \\
& +\frac{\xi}{v_{1}} \int_{0}^{w} f^{2} d s,
\end{aligned}
$$

$$
\text { where } v_{1}>0 \text {. Combining (11)-(16), we derive } \dot{\Theta}_{2}(t) \text { as }
$$

$$
\begin{aligned}
\dot{\Theta}_{2}(t)= & \sigma x(t) \tau_{e}(t)-\sigma x(t) \\
& \times\left[\widetilde{T} p^{\prime}(w, t)-\widetilde{E} I p^{\prime \prime \prime}(w, t)\right. \\
& \left.\quad+\widetilde{d}_{a} \dot{p}(w, t)+\widetilde{m}\left(\kappa_{2} \dot{p}^{\prime \prime \prime}(w, t)-\kappa_{3} \dot{p}^{\prime}(w, t)\right)\right] \\
& -\sigma x(t) \tanh (x(t)) \hat{D}+\sigma x(t) d(t)-\sigma \kappa_{1} x^{2}(t) .
\end{aligned}
$$

Invoking (17)-(24), we obtain $\dot{\Theta}_{3}(t)$ as

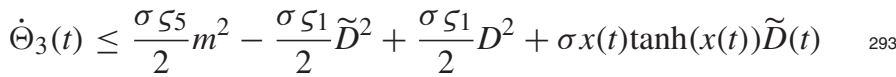

$$
\begin{aligned}
& +\sigma x(t)\left[\widetilde{T} p^{\prime}(w, t)-\widetilde{E} I p^{\prime \prime \prime}(w, t)+\widetilde{d}_{a} \dot{p}(w, t)\right. \\
& \left.+\widetilde{m}\left(\kappa_{2} \dot{p}^{\prime \prime \prime}(w, t)-\kappa_{3} \dot{p}^{\prime}(w, t)\right)\right]-\frac{\sigma \varsigma_{2}}{2} \widetilde{T}^{2}-\frac{\sigma \varsigma 3}{2} \widetilde{E} I^{2}
\end{aligned}
$$

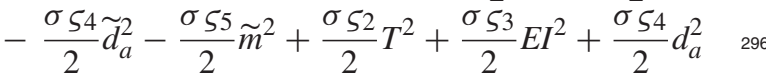

$$
\begin{aligned}
& -\sigma \varrho \tilde{\lambda} \beta \alpha(t) x(t)-\frac{\sigma v}{2} \tilde{\lambda}^{2}+\frac{\sigma v}{2} \lambda^{2} \text {. }
\end{aligned}
$$

We invoke (1) and apply Lemma 1 to derive $\dot{\Theta}_{4}(t)$ as 298

$$
\begin{aligned}
\dot{\Theta}_{4}(t) \leq & -w \psi E I p^{\prime \prime \prime}(w, t) p^{\prime}(w, t)-\frac{3 \psi E I}{2} \int_{0}^{w} p^{\prime \prime 2} d s \\
& +\frac{w \psi c}{\nu_{2}} \int_{0}^{w} \dot{p}^{2} d s+\frac{\psi \rho w}{2} \dot{p}^{2}(w, t)+\frac{w \psi}{v_{3}} \int_{0}^{w} f^{2} d s \\
& -\left(\frac{\psi T}{2}-\psi v_{2} c w-\psi v_{3} w\right) \int_{0}^{w} p^{\prime 2} d s \\
& +\frac{\psi T w}{2} p^{\prime 2}(w, t)-\frac{\psi \rho}{2} \int_{0}^{w} \dot{p}^{2} d s
\end{aligned}
$$

where $v_{2}, v_{3}>0$.
303 
304 Substituting (37)-(40) into (36) and using Lemmas 1-3 305 and $(13), \dot{\Theta}(t)$ gives

$$
\begin{aligned}
& 306 \quad \dot{\Theta}(t) \leq-\frac{3 \psi E I}{2} \int_{0}^{w} p^{\prime \prime 2} d s \\
& -\left(\frac{\xi E I \kappa_{3}^{2}}{2 \kappa_{2}}-\frac{\xi\left|T-E I \kappa_{3} / \kappa_{2}\right|}{2 v_{4}}\right. \\
& \left.-\frac{E I\left|\xi \kappa_{3}-w \psi\right| \nu_{5}}{2}-\frac{\psi T w}{2}\right) p^{\prime 2}(w, t)-\frac{\sigma \zeta_{1}}{2} \widetilde{D}^{2} \\
& -\left(\frac{\xi E I}{2 \kappa_{2}}-\frac{\xi\left|T-E I \kappa_{3} / \kappa_{2}\right| v_{4}}{2}-\frac{\psi \rho w}{2}\right) \dot{p}^{2}(w, t) \\
& -\left(\frac{\xi E I \kappa_{2}}{2}-\frac{\left|\xi \kappa_{3}-w \psi\right|}{2 \nu_{5}}\right) p^{\prime \prime \prime 2}(w, t)+\frac{\sigma \varsigma_{1}}{2} D^{2} \\
& -\left(\xi c-\xi \nu_{1}-\frac{w \psi c}{\nu_{2}}+\frac{\psi \rho}{2}\right) \int_{0}^{w} \dot{p}^{2} d s+\frac{\sigma \varsigma_{5}}{2} m^{2} \\
& -\left(\frac{\psi T}{2}-\psi v_{2} c w-\psi v_{3} w\right) \int_{0}^{w} p^{\prime 2} d s-\frac{\sigma \varsigma_{1}}{2} \widetilde{D}^{2} \\
& -\left(\sigma \kappa_{1}-\frac{\xi E I}{2 \kappa_{2}}\right) x^{2}(t)-\frac{\sigma \varsigma_{2}}{2} \widetilde{T}^{2}-\frac{\sigma \varsigma_{3}}{2} \widetilde{E I}^{2}+0.2785 \sigma D
\end{aligned}
$$

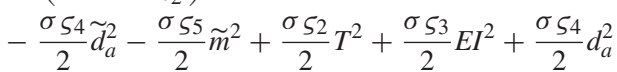

$$
\begin{aligned}
& +\left(\frac{\xi}{v_{1}}+\frac{w \psi}{v_{3}}\right) \int_{0}^{w} f^{2} d s-\frac{\sigma v}{2} \tilde{\lambda}^{2}+\frac{\sigma v}{2} \lambda^{2}
\end{aligned}
$$

316 where $v_{4}, v_{5}>0$ and we choose $\psi, \sigma, \xi, \kappa_{i}, i=1 \cdots 3, v_{j}$, 317 for $j=1 \cdots 5$ to satisfy

$$
\begin{aligned}
& { }_{318} \quad \frac{\xi E I \kappa_{3}^{2}}{2 \kappa_{2}}-\frac{\xi\left|T-E I \kappa_{3} / \kappa_{2}\right|}{2 \nu_{4}}-\frac{E I\left|\xi \kappa_{3}-w \psi\right| \nu_{5}}{2}-\frac{\psi T w}{2} \geq 0 \\
& 319 \\
& 20 \\
& \frac{\xi E I}{2 \kappa_{2}}-\frac{\xi\left|T-E I \kappa_{3} / \kappa_{2}\right| \nu_{4}}{2}-\frac{\psi \rho w}{2} \geq 0 \\
& \frac{\xi E I \kappa_{2}}{2}-\frac{\left|\xi \kappa_{3}-w \psi\right|}{2 \nu_{5}} \geq 0 \\
& \omega_{1}=\xi c-\xi v_{1}-\frac{w \psi c}{v_{2}}+\frac{\psi \rho}{2}>0 \\
& \omega_{2}=\frac{\psi T}{2}-\psi v_{2} c w-\psi \nu_{3} w>0 \\
& \omega_{3}=\sigma \kappa_{1}-\frac{\xi E I}{2 \kappa_{2}}>0 \\
& \chi=\left(\frac{\xi}{v_{1}}+\frac{w \psi}{\nu_{3}}\right) w F^{2}+\frac{\sigma \varsigma_{1}}{2} D^{2}+\frac{\sigma \varsigma_{2}}{2} T^{2}+\frac{\sigma \varsigma_{3}}{2} E I^{2} \\
& +\frac{\sigma \varsigma_{4}}{2} d_{a}^{2}+\frac{\sigma \varsigma_{5}}{2} m^{2}+\frac{\sigma v}{2} \varepsilon^{2}+0.2785 \sigma D<+\infty .
\end{aligned}
$$

Combining (42)-(48), (41) is derived as

$$
\begin{aligned}
& \dot{\Theta}(t) \leq \chi-\frac{\sigma \varsigma_{1}}{2} \widetilde{D}^{2}-\omega_{1} \int_{0}^{w} \dot{p}^{2} d s-\omega_{2} \int_{0}^{w} p^{\prime 2} d s \\
& -\frac{3 \psi E I}{2} \int_{0}^{w} p^{\prime \prime 2} d s-\omega_{3} x^{2}(t)-\frac{\sigma v}{2} \widetilde{\lambda}^{2} \\
& -\frac{\sigma \varsigma_{2}}{2} \widetilde{T}^{2}-\frac{\sigma \varsigma_{3}}{2} \widetilde{E} I^{2}-\frac{\sigma \varsigma_{4}}{2} \widetilde{d}_{a}^{2}-\frac{\sigma \varsigma_{5}}{2} \widetilde{m}^{2} \\
& \leq-\iota_{3}\left[\Theta_{1}(t)+\Theta_{2}(t)+\Theta_{3}(t)\right]+\chi
\end{aligned}
$$

333 where $\iota_{3}=\min \left(\frac{2 \omega_{1}}{\xi \rho}, \frac{2 \omega_{2}}{\xi T}, \frac{3 \psi}{\xi}, \frac{2 \omega_{3}}{\sigma m}, \varsigma_{1}, \varsigma_{2}, \varsigma_{3}, \varsigma_{4}, \varsigma_{5}, 1\right)$.

334 We then invoke (30) and (49) to obtain

$$
{ }_{335} \dot{\Theta}(t) \leq-\iota \Theta(t)+\chi
$$

336 where $\iota=\left(\iota_{3} / \iota_{2}\right)$.

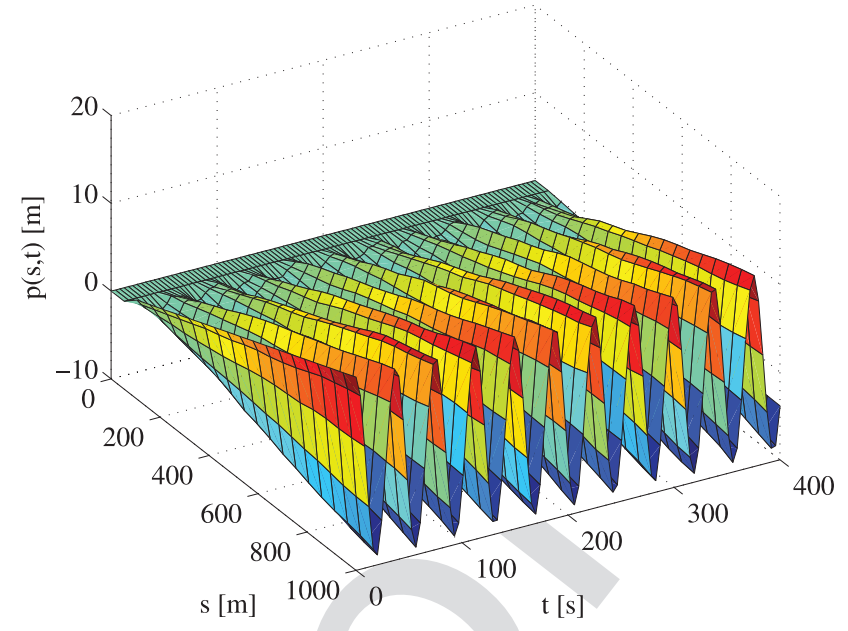

Fig. 3. 3-D offset of the riser under no control.

Theorem 1: For the riser system with input backlash (4), 337 under the presented adaptive backlash inverse control (15), ззв online updating laws (18)-(23), and bounded initial conditions, with the choice of design parameters $\psi, \sigma, \xi, \kappa_{i}, i=1 \cdots 3, v_{j}, 340$ for $j=1 \cdots 5$ satisfying constraints (42)-(48), we arrive ${ }_{341}$ at a conclusion that the controlled system's state $p(s, t)$ is 342 uniformly ultimately bounded.

Proof: We multiply (35) by $e^{\imath t}$ and then integrate the ${ }_{344}$ consequence to derive

$$
\Theta(t) \leq \Theta(0) e^{-\iota t}+\frac{\chi}{\iota}\left(1-e^{-\imath t}\right) \leq \Theta(0) e^{-\imath t}+\frac{\chi}{\iota} .
$$

Invoking $\Theta_{1}(t),(30)$, and Lemma 2, we get

$$
\frac{\xi T}{2 w} p^{2}(s, t) \leq \frac{\xi T}{2} \int_{0}^{w} p^{\prime 2}(s, t) d s \leq \Theta_{1}(t) \leq \frac{1}{\iota_{1}} \Theta(t) .
$$

We substitute (51) into (52) to derive

$$
|p(s, t)| \leq \sqrt{\frac{2 w}{\xi \iota_{1} T}\left[\Theta(0) e^{-\imath t}+\frac{\chi}{\iota}\right]}, \forall(s, t) \in[0, w] \times[0,+\infty) .
$$

Combining (53) further gives

$$
352
$$$$
\lim _{t \rightarrow \infty}|p(s, t)| \leq \sqrt{\frac{2 w \chi}{\xi T \iota_{1} \iota}}, \forall s \in[0, w] .
$$

Remark 2. This article presents a framework of adaptive inverse control of uncertain vessel-riser systems subject 356 to input backlash, system uncertainties, and external distur- 357 bances, which is invalid for the system with input hysteresis. 358 To address this issue, the approaches in [50] will be employed 359 in the next step. Moreover, an issue about communication limit 360 in actuator is ignored in this article, and we will cope with it 361 with recourse to the technique in [69]-[71].

362

\section{Numerical Simulation}

On the basis of the vessel-riser system dynamical model 364 with input backlash (1), (2), (4), and (13), we exploit 365 the finite difference method [72] with time and space 366 


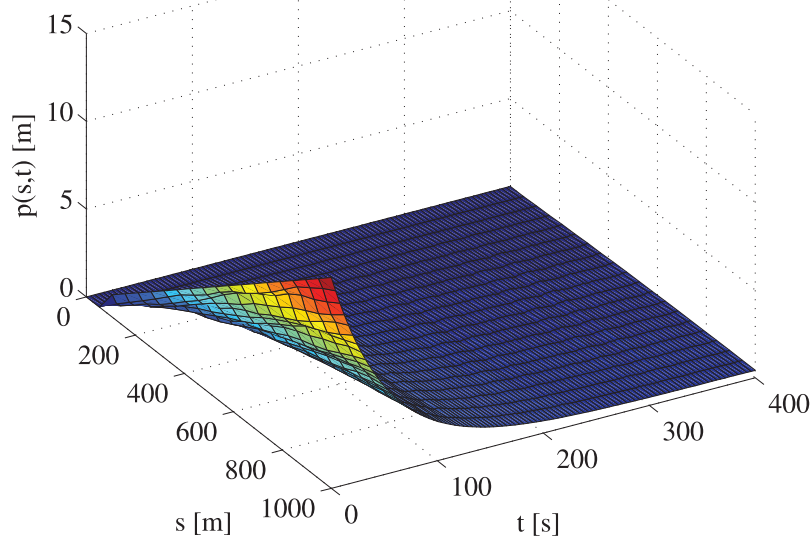

Fig. 4. 3-D offset of the riser under proposed control.

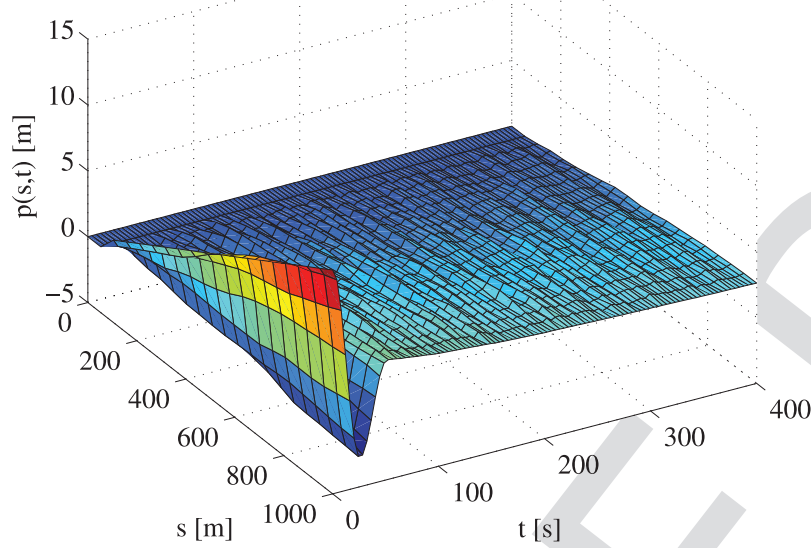

Fig. 5. 3-D offset of the riser under previous control in [18].

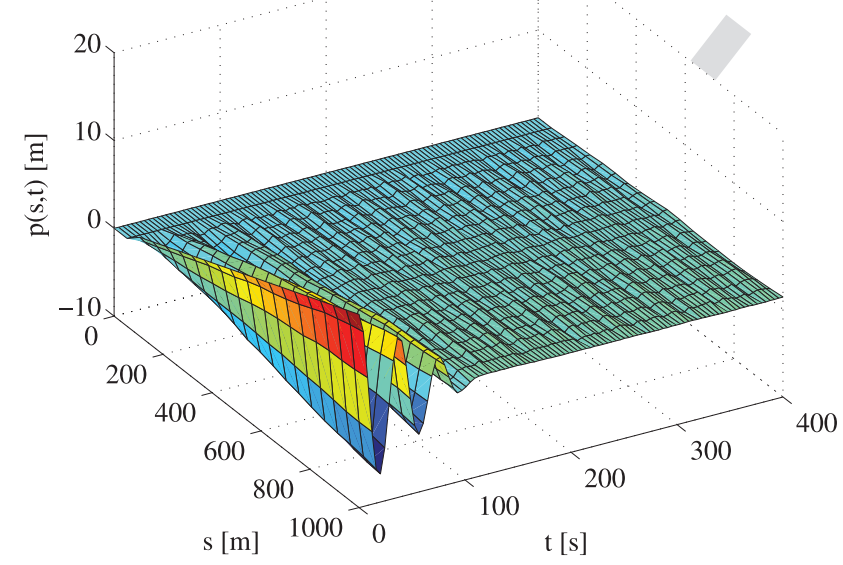

Fig. 6. 3-D offset of the riser under previous control in [60].

367 steps as $2 \times 10^{-5}$ and 0.05 to illustrate the dynam368 ics of this nonlinear coupled system by setting system ${ }_{369}$ parameters as $E I=1.5 \times 10^{7} \mathrm{Nm}^{2}, \rho=500 \mathrm{~kg} / \mathrm{m}$,

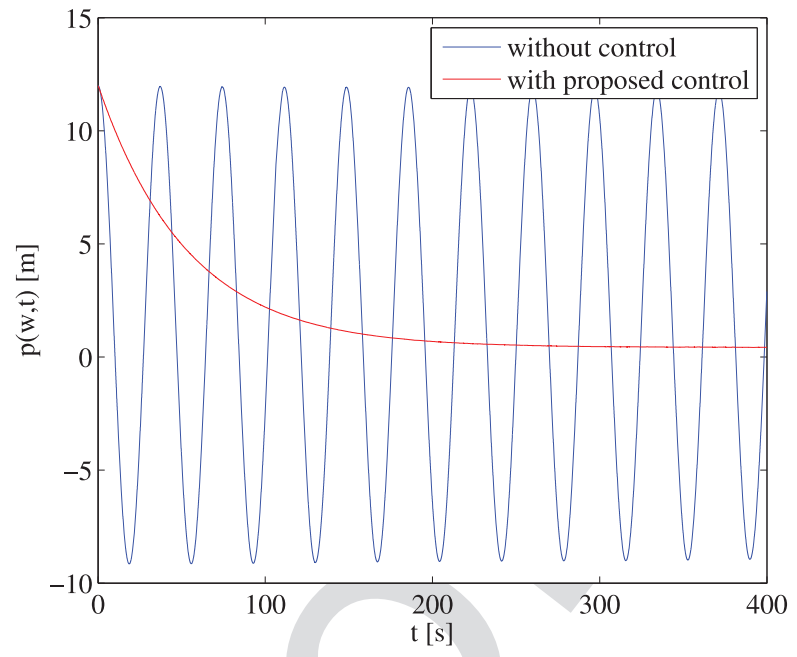

Fig. 7. 2-D offset of the vessel under proposed control.

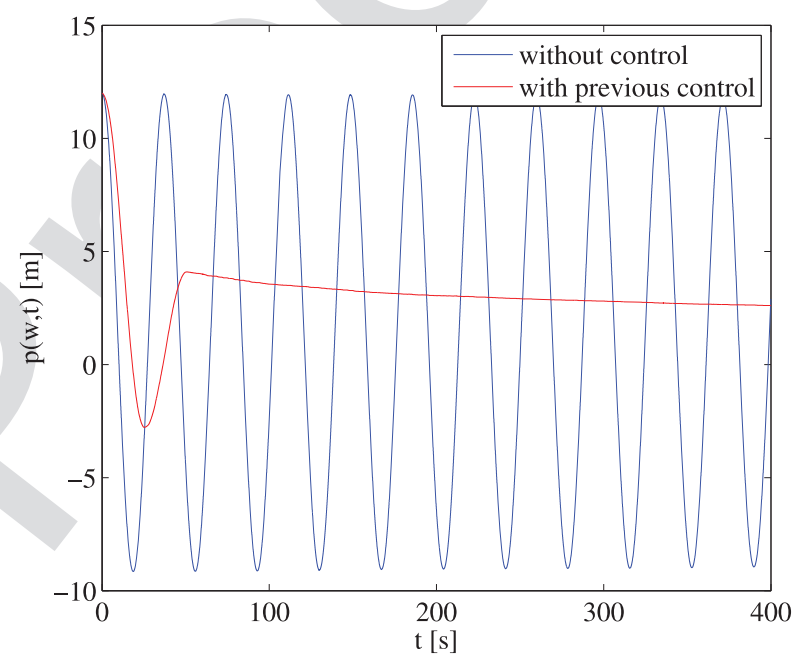

Fig. 8. 2-D offset of the vessel under previous control in [18].

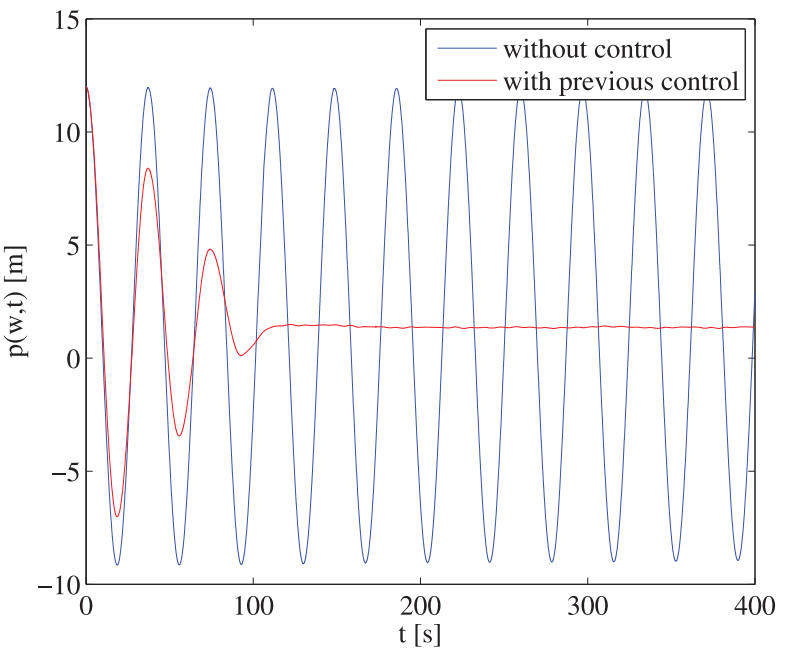

Fig. 9. 2-D offset of the vessel under previous control in [60].

$T=3.0 \times 10^{8} \mathrm{~N}, c=1.0 \mathrm{Ns} / \mathrm{m}^{2}, w=1000 \mathrm{~m}, d_{a}=370$ $1.5 \times 10^{5} \mathrm{Ns} / \mathrm{m}$, and $m=9.6 \times 10^{6} \mathrm{~kg}$. System initial condi- 371 tions are presented as $p(s, 0)=12 \sin \left(\frac{s}{w}\right)$ and $\dot{p}(s, 0)=0 . \quad 372$ 


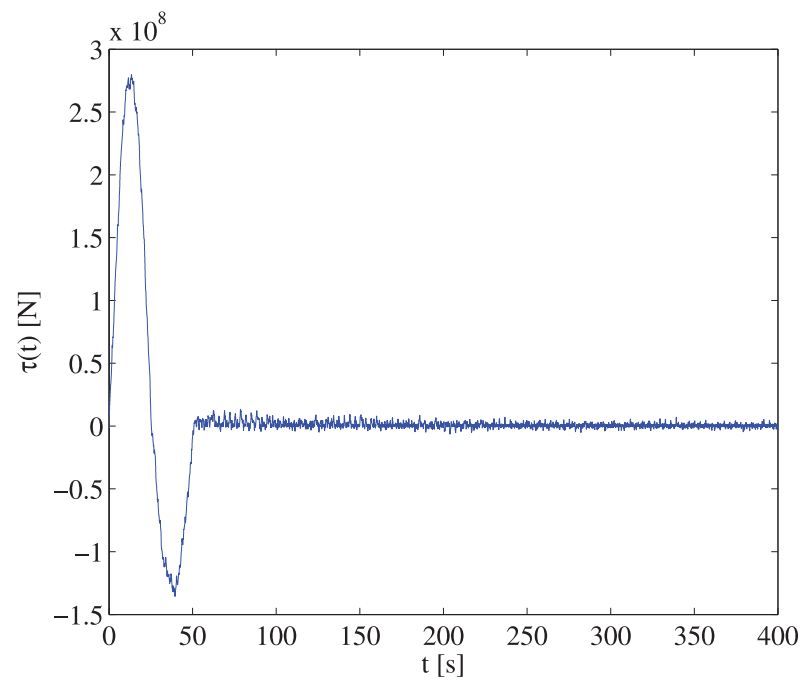

Fig. 10. Previous control input in [18].

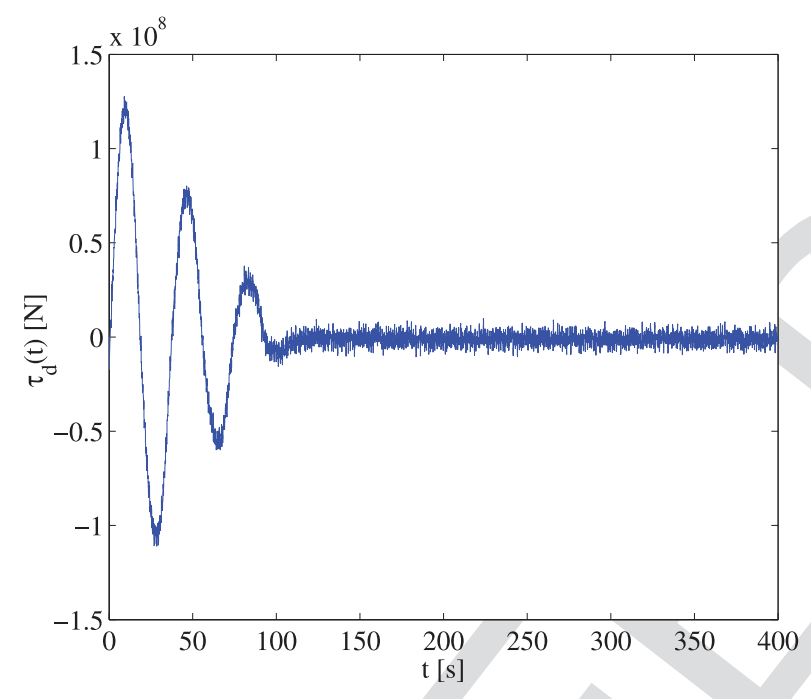

Fig. 11. Designed control command in [60].

373 Meanwhile, $d(t)$ is given as $d(t)=[3+0.8 \sin (0.7 t)+$ $3740.2 \sin (0.5 t)+0.2 \sin (0.9 t)] \times 10^{5}$.

375 Without any control, namely, $\tau(t)=0$, 3-D and two376 dimensional (2-D) responses of this coupled system are 377 portrayed in Figs. 3 and 7. From Fig. 3, it is seen that 378 the marine flexible riser is vibrating with an equal ampli379 tude under the external ocean disturbance. Fig. 7 illus380 trates the displacement of the vessel in the ocean surface. 381 The persistent large deformation of this marine riser will 382 lead to the produce fatigue problems, and it is crucial to 383 reduce the vibration by implementing the effective control 384 strategy.

${ }_{385}$ With presented control law (15) by choosing control gains ${ }_{386} \kappa_{1}=3 \times 10^{8}, \kappa_{2}=1, \kappa_{3}=20$, and control parameters ${ }_{387} \varsigma_{1}=\varsigma_{2}=\varsigma_{3}=\varsigma_{4}=\varsigma_{5}=0.001, \varrho=1, \beta=1.2$, ${ }_{388} v=3, B=3 \times 10^{6}$, and $B_{m}=1 \times 10^{7}$, the spatio-temporal 389 response and end point offset are depicted in Figs. 4 and 7. ${ }_{390}$ From Figs. 4 and 7, it is seen that the effects of the considered

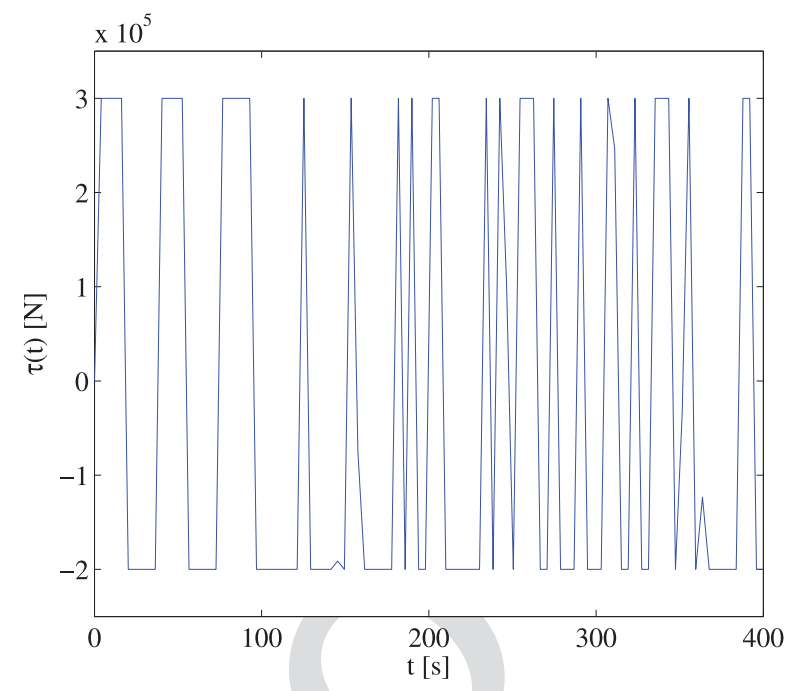

Fig. 12. Saturation control input in [60].

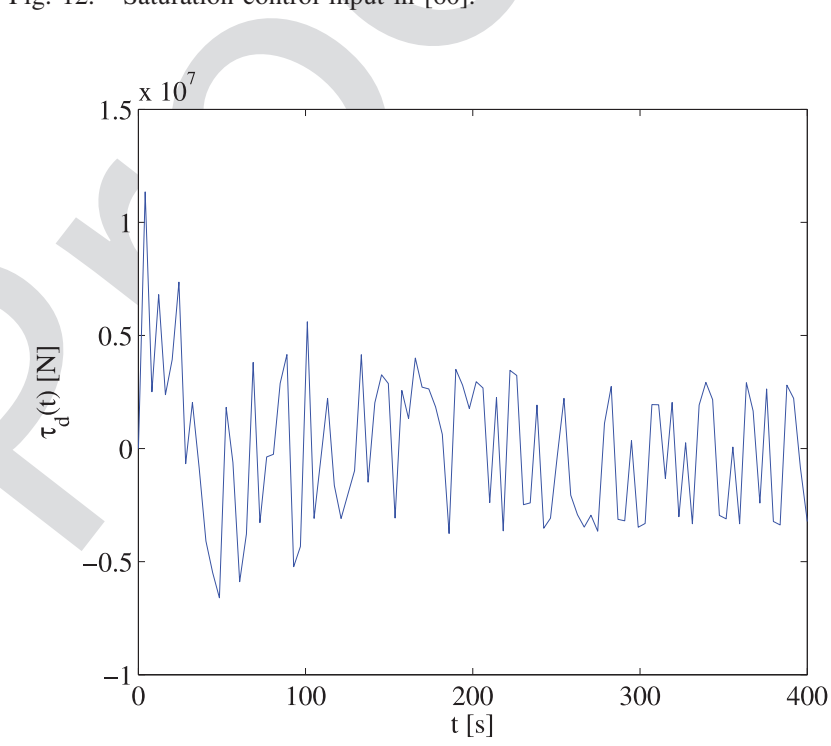

Fig. 13. Designed control input.

external ocean disturbance and the input backlash nonlinearity 391 are eliminated under the proposed control (15). Moreover, it 392 has a positive effect on the vibration attenuation of the marine 393 flexible riser, and the displacement of the vessel reduces to a 394 small neighborhood around the original position. Meanwhile, 395 Figs. 13 and 14 display 2-D responses of presented control 396 input and backlash input.

For the comparison with the proposed control law, we con- 398 sider two control strategies presented in previous works [18] 399 and [60]. When exerting the previous control proposed in [18] 400 on the riser system with the given control parameters $k={ }_{401}$ $3 \times 10^{8}, k_{1}=1$, and $k_{2}=10$, Figs. 5, 8, and 10 dis- 402 play the responses of the marine flexible riser, vessel, and ${ }_{403}$ the control law, respectively. Note that this previous research 404 does not consider the effect of the input constraint and 405 the control law presented in [18] requires longer convergent 406 time and larger convergent neighborhood than the proposed ${ }_{407}$ control (15) for the marine vessel-riser system with input 408 


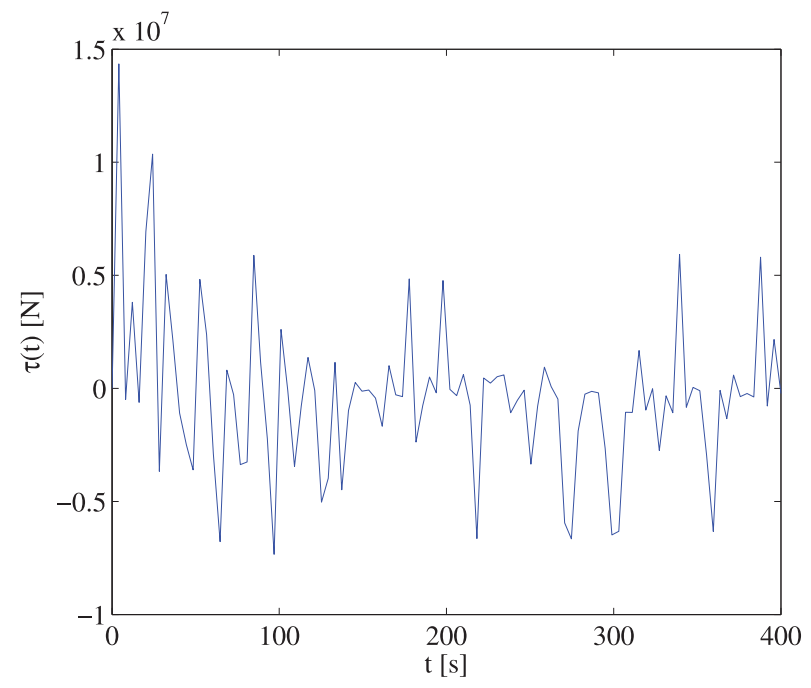

Fig. 14. Backlash control input.

409 backlash nonlinearity. Under the action of previous anti410 saturation control developed in [60] on the riser system, when ${ }_{411}$ the control design parameters are selected as $k_{1}=k_{3}=\beta_{1}=$ ${ }_{412} \beta_{2}=\beta_{3}=\beta_{4}=1, k_{2}=20, k_{4}=0.01, k_{5}=8 \times 10^{7}$, ${ }_{413} \sigma_{1}=\sigma_{2}=\sigma_{3}=\sigma_{4}=1 \times 10^{-4}, \sigma=1 \times 10^{-5}, \tau_{\max }=3 \times 10^{5}$, ${ }_{414}$ and $\tau_{\min }=-2 \times 10^{5}$, the time and spatial responses are 415 described in Figs. 6, 9, 11, and 12. Note that the control 416 law presented in [60] also has the positive effect on reduc417 ing the deformation of the coupled system, however it has 418 the large overshoot and convergent neighborhood than the 419 proposed control (15).

420 We observe from Figs. 3-14 that the vibration in the cou421 pled vessel-riser system is observably suppressed under the 422 proposed adaptive inverse control, which achieves a better con423 trol performance than the previous control; the end point offset ${ }_{424} p(w, t)$ is stabilized at a small region around zero, and the 425 backlash nonlinearity in the control input is fairly obvious. In 426 other words, this approach leads to a good performance on 427 the vibration decrease, uncertainties compensation, and input 428 backlash elimination.

\section{$429 \quad$ V. CONCLUSION}

430 The framework of the adaptive inverse control of uncer431 tain vessel-riser systems possessing input backlash has been ${ }_{432}$ presented in this article. The adaptive inverse of backlash ${ }_{433}$ was used to formulate the nonlinear input backlash as a ${ }_{434}$ desired control signal with a mismatch error. An adaptive 435 inverse control and relevant adaptive laws were presented for ${ }_{436}$ stabilizing the riser's offset, eliminating the backlash, and 437 compensating for system uncertainties. Exploiting the rigor438 ous analysis without recourse to model reducing technique, 439 the derived control ensured and realized the uniform stabil440 ity of the controlled system. In conclusion, the simulation 441 comparison studies validated the control performance. Future 442 interesting topics include exploiting the intelligent control 443 techniques [73]-[79] to regulate the transient performance of 444 the controlled vessel-riser systems.

\section{REFERENCES}

[1] S.-L. Dai, S. D. He, M. Wang, and C. Z. Yuan, "Adaptive neural 446 control of underactuated surface vessels with prescribed performance 447 guarantees," IEEE Trans. Neural Netw. Learn. Syst., to be published. 448 doi: 10.1109/TNNLS.2018.2876685.

[2] N. Wang, J.-C. Sun, M. Han, Z. J. Zheng, and M. J. Er, "Adaptive 450 approximation-based regulation control for a class of uncertain nonlin- 451 ear systems without feedback linearizability," IEEE Trans. Neural Netw. 452 Learn. Syst., vol. 29, no. 8, pp. 3747-3760, Aug. 2018.

[3] J. L. Li, Q. M. Yang, B. Fan, and Y. X. Sun, "Robust state/output- 454 feedback control of coaxial-rotor MAVs based on adaptive NN 455 approach," IEEE Trans. Neural Netw. Learn. Syst., to be published. 456 doi: 10.1109/TNNLS.2019.2911649.

[4] S.-L. Dai, C. Wang, and M. Wang, "Dynamic learning from adaptive 458 neural network control of a class of nonaffine nonlinear systems," IEEE 459 Trans. Neural Netw. Learn. Syst., vol. 25, no. 1, pp. 111-123, Jan. 2014. 460

[5] W. He and Y. Dong, "Adaptive fuzzy neural network control for a con- 461 strained robot using impedance learning," IEEE Trans. Neural Netw. 462 Learn. Syst., vol. 29, no. 4, pp. 1174-1186, Apr. 2018.

[6] Z. Li, J. Li, and Y. Kang, "Adaptive robust coordinated control of 464 multiple mobile manipulators interacting with rigid environments," 465 Automatica, vol. 46, no. 12, pp. 2028-2034, 2010.

[7] B. Niu, Y. J. Liu, W. L. Zhou, H. T. Li, P. Y. Duan, and J. Q. Li, 467 "Multiple Lyapunov functions for adaptive neural tracking control of 468 switched nonlinear non-lower-triangular systems," IEEE Trans. Cybern., 469 to be published. doi: 10.1109/TCYB.2019.2906372.

[8] B. Niu, P. Y. Duan, J. Q. Li, and X. D. Li, "Adaptive neural tracking 471 control scheme of switched stochastic nonlinear pure-feedback non- 472 lower triangular systems," IEEE Trans. Syst., Man, Cybern., Syst., to 473 be published. doi: 10.1109/TSMC.2019.2894745.

[9] C. G. Yang, Y. M. Jiang, J. Na, Z. J. Li, and B. Xu, "Adaptive 475 parameter estimation and control design for robot manipulators with 476 finite-time convergence," IEEE Trans. Ind. Electron., vol. 65, no. 10, 477 pp. 8112-8123, Oct. 2018.

[10] H. Y. Li, S. Y Zhao, W. He, and R. Q. Lu, "Adaptive finite-time tracking control of full states constrained nonlinear systems with dead-zone," 480 Automatica, vol. 100, pp. 99-107, Feb. 2019.

[11] J. Fu, R. C. Ma, and T. Y Chai, "Adaptive finite-time stabilization of a 482 class of uncertain nonlinear systems via logic-based switchings," IEEE 483 Trans. Autom. Control, vol. 62, no. 11, pp. 5998-6003, Nov. 2017. 484

[12] Y.-J. Liu, S. M. Lu, S. C. Tong, X. K. Chen, C. L. P. Chen, and 485 D.-J. Li, "Adaptive control-based barrier Lyapunov functions for a class 486 of stochastic nonlinear systems with full state constraints," Automatica, 487 vol. 87, pp. 83-93, Jan. 2018

[13] S. Zhang, Y. T. Dong, Y. C. Ouyang, Z. Yin, and K. X. Peng, "Adaptive 489 neural control for robotic manipulators with output constraints and 490 uncertainties," IEEE Trans. Neural Netw. Learn. Syst., vol. 29, no. 11, 491 pp. 5554-5564, Nov. 2018.

[14] Y.-J. Liu, M. Z. Gong, S. C. Tong, C. L. P. Chen, and D.-J. Li, 493 "Adaptive fuzzy output feedback control for a class of nonlinear systems 494 with full state constraints," IEEE Trans. Fuzzy Syst., vol. 26, no. 5, 495 pp. 2607-2617, Oct. 2018.

[15] Y. Y. Wang, H. Shen, H. R. Karimi, and D. P. Duan, "Dissipativity- 497 based fuzzy integral sliding mode control of continuous-time T-S fuzzy 498 systems," IEEE Trans. Fuzzy Syst., vol. 26, no. 3, pp. 1164-1176, 499 Jun. 2018.

[16] Y. Y. Wang, H. R. Karimi, H. Shen, Z. J. Fang, and M. X. Liu, "Fuzzy- 501 model-based sliding mode control of nonlinear descriptor systems," 502 IEEE Trans. Cybern., vol. 49, no. 9, pp. 3409-3419, Sep. 2019.

[17] K.-S. Hong and U. H. Shah, "Vortex-induced vibrations and control of 504 marine risers: A review," Ocean Eng., vol. 152, pp. 300-315, Mar. 2018. 505

[18] W. He, S. S. Ge, B. V. E. How, Y. S. Choo, and K.-S. Hong, 506 "Robust adaptive boundary control of a flexible marine riser with vessel 507 dynamics," Automatica, vol. 47, no. 4, pp. 722-732, 2011.

[19] M. Zapateiro, N. Luo, H. R. Karimi, and J.Vehí, "Vibration control 509 of a class of semiactive suspension system using neural network and 510 backstepping techniques," Mech. Syst. Signal Process., vol. 23, no. 6, 511 pp. 1946-1953, 2009.

20] M. Zapateiro, F. Pozo, H. R. Karimi, and N. S. Luo, "Semiactive 513 control methodologies for suspension control with magnetorheological 514 dampers," IEEE/ASME Trans. Mechatronics, vol. 17, no. 2, pp. 370-380, 515 Apr. 2012.

[21] M. L. Wang, H.-X. Li, X. Chen, and Y. Chen, "Deep learning-based 517 model reduction for distributed parameter systems," IEEE Trans. Syst., 518 Man, Cybern., Syst., vol 46, no. 12, pp. 1664-1674, Dec 2016.

\section{.} .

.

3

5

.

(1)

1
463
464 465
466
467 68

(4)

472
473
474

474

6

478

979

49

80
81

82

4

487

8

0

49


22] C. Sun, H. Gao, W. He, and Y Yu, "Fuzzy neural network control of a flexible robotic manipulator using assumed mode method," IEEE Trans. Neural Netw. Learn. Syst., vol. 29, no. 11, pp. 5214-5227, Nov. 2018.

[23] H.-N. Wu and J.-W. Wang, "Static output feedback control via PDE boundary and ODE measurements in linear cascaded ODE-beam systems," Automatica, vol. 50, no. 11, pp. 2787-2798, 2014.

[24] Z. J. Liu, J. K. Liu, and W. He, "Robust adaptive fault tolerant control for a linear cascaded ODE-beam systems," Automatica, vol. 98, pp. 42-50, Dec. 2018.

[25] U. H. Shah and K.-S. Hong, "Active vibration control of a flexible rod moving in water: Application to nuclear refueling machines," Automatica, vol. 93, pp. 231-243, Jul. 2018.

[26] J.-M. Wang, J.-J. Liu, B. B. Ren, and J. H. Chen, "Sliding mode control to stabilization of cascaded heat PDE-ODE systems subject to boundary control matched disturbance," Automatica, vol. 52, pp. 23-34, Feb. 2015

[27] T. Endo, F. Matsuno, and Y. Jia, "Boundary cooperative control by flexible Timoshenko arms," Automatica, vol. 81, pp. 377-389, Jul. 2017.

[28] L. Su, J.-M. Wang, and M. Krstic, "Feedback stabilization of a class of coupled hyperbolic equations with non-local terms," IEEE Trans. Autom. Control, vol. 63, no. 8, pp. 2633-2640, Aug. 2018.

[29] Z. J. Liu, J. K. Liu, and W. He, "Dynamic modeling and vibration control for a nonlinear 3-dimensional flexible manipulator," Int. J. Robust Nonlin. Control, vol. 28, no. 13, pp. 3927-3945, 2018.

[30] W. He and S. S. Ge, "Cooperative control of a nonuniform gantry crane with constrained tension" Automatica, vol. 66, no. 4, pp. 146-154, 2016.

55 [31] Z. J. Zhao, C. K. Ahn, and H.-X. Li, "Boundary antidisturbance control of a spatially nonlinear flexible string system," IEEE Trans. Ind. Electron., to be published. doi: 10.1109/TIE.2019.2931230.

[32] F.-F. Jin and B.-Z. Guo, "Lyapunov approach to output feedback stabilization for the Euler-Bernoulli beam equation with boundary input disturbance," Automatica, vol. 52, pp. 95-102, Feb. 2015

[33] J.-W. Wang, Y.-Q. Liu, and C.-Y. Sun, "Adaptive neural boundary control design for nonlinear flexible distributed parameter systems," IEEE Trans. Control Syst. Technol., vol. 27, no. 5, pp. 2085-2099, Sep. 2019. doi: 10.1109/TCST.2018.2849072.

[34] Y. Orlov, A. Pisano, and E. Usai, "Exponential stabilization of the uncertain wave equation via distributed dynamic input extension," IEEE Trans. Autom. Control, vol. 56, no. 1, pp. 212-217, Jan. 2011.

[35] M. Dogan and Ö. Morgül, "On the control of two-link flexible robo arm with nonuniform cross section," J. Vib. Control, vol. 16, no. 5 , pp. 619-646, 2010

[36] X. Y. He, W. He, J. Shi, and C. Y. Sun, "Boundary vibration control of variable length crane systems in two dimensional space with output constraints," IEEE/ASME Trans. Mechatronics, vol. 22, no. 5, pp. 1952-1962, Oct. 2017

[37] A. Tavasoli, "Adaptive robust boundary control of shaft vibrations under perturbations with unknown upper bounds," Int. J. Adapt. Control Signal Process., vol. 29, no. 5, pp. 537-562, 2015

[38] X. Cai and M. Krstic, "Nonlinear stabilization through wave PDE dynamics with a moving uncontrolled boundary," Automatica, vol. 68 , pp. 27-38, Jun. 2016

1 [39] W. He, Y. Ouyang, and J. Hong, "Vibration control of a flexible robotic manipulator in the presence of input deadzone," IEEE Trans. Ind. Informat., vol. 13, no. 1, pp. 48-59, Feb. 2017

4 [40] C. Y. Sun, W. He, and J. Hong, "Neural network control of a flexible robotic manipulator using the lumped spring-mass model," IEEE Trans. Syst., Man, Cybern., Syst., vol. 47, no. 8, pp. 1863-1874, Aug. 2017.

[41] Z.-P. Wang, H.-N. Wu, and H.-X. Li, "Estimator-based $H_{\infty}$ sampled-dat fuzzy control for nonlinear parabolic PDE systems," IEEE Trans. Syst. Man, Cybern., Syst., to be published. doi: 10.1109/TSMC.2018.2818761.

[42] F. Guo, Y. Liu, Y. L. Wu, and F. Luo, "Observer-based backstepping boundary control for a flexible riser system," Mech. Syst. Signal Process. vol. 111, pp. 314-330, Oct. 2018

[43] Z. J. Zhao, Y. Liu, and F. Guo, "Robust output feedback stabilization for a flexible marine riser system," ISA Trans., vol. 78, pp. 130-140, Jul. 2018.

6 [44] K. D. Do, "Boundary control design for extensible marine risers in three dimensional space," J. Sound Vib., vol. 388, no. 3, pp. 1-19, 2017.

8 [45] K. D. Do and A. D. Lucey, "Boundary stabilization of extensible and unshearable marine risers with large in-plane deflection," Automatica, vol. 77, pp. 279-292, Mar. 2017.

[46] K. D. Do, "Stochastic boundary control design for extensible marin risers in three dimensional space," Automatica, vol. 77, pp. 184-197, Mar. 2017.

[47] M. Chen and G. Tao, "Adaptive fault-tolerant control of uncertain nonlinear large-scale systems with unknown dead-zone," IEEE Trans. Cybern. vol. 46, no. 8, pp. 1851-1862, Aug. 2016.
[48] W. Kang and M. Fridman, "Boundary control of delayed ODE-heat 597 cascade under actuator saturation," Automatica, vol. 83, pp. 252-261, 598 Sep. 2017.

[49] M. Chen, P. Shi, and C.-C. Lim, "Robust constrained control for MIMO 600 nonlinear systems based on disturbance observer," IEEE Trans. Autom. 60 Control, vol. 60, no. 12, pp. 3281-3286, Dec. 2015.

[50] J. Zhou and C. Wen, Adaptive Backstepping Control of Uncertain Systems: Nonsmooth Nonlinearities, Interactions or Time-Variations. 604 Berlin, Germany: Springer, 2008

[51] W. He, Z Li, Y. Dong, and T. Zhao, "Design and adaptive control for an upper limb robotic exoskeleton in presence of input saturation," IEEE 607 Trans. Neural Netw. Learn. Syst., vol. 30, no. 1, pp. 97-108, Jan. 2019. 608

[52] W. He, Y. Sun, Z. Yan, C. Yang, Z. Li, and O. Kaynak, "Disturbance 609 observer-based neural network control of cooperative multiple manipu- 610 lators with input saturation," IEEE Trans. Neural Netw. Learn. Syst., to 611 be published. doi: 10.1109/TNNLS.2019.2923241.

[53] Z. Li, Z. Chen, J. Fu, and C. Sun, "Direct adaptive controller for uncer- 613 tain MIMO dynamic systems with time-varying delay and dead-zone 614 inputs," Automatica, vol. 63, pp. 287-291, Jan. 2016.

[54] W. C. Meng, Q. M. Yang, S. Jagannathan, and Y. X. Sun, "Distributed 61 control of high-order nonlinear input constrained multiagent systems ${ }_{617}$ AQ3 using a backstepping-free method," IEEE Trans. Cybern., vol. 49, no. 11, 618 pp. 3923-3933, Nov. 2019. doi: 10.1109/TCYB.2018.2853623.

[55] H. Wang, H. R. Karimi, P. X. Liu, and H. Yang, "Adaptive neural con- 620 trol of nonlinear systems with unknown control directions and input 621 dead-zone," IEEE Trans. Syst., Man, Cybern., Syst., vol. 48, no. 11, 622 pp. 1897-1907, Nov. 2018

56] M. Chen, Y Ren, and J. Liu, "Anti-disturbance control for a suspension 624 cable system of helicopter subject to input nonlinearities," IEEE Trans. 625 Syst., Man, Cybern., Syst., vol. 48, no. 12, pp. 2292-2304, Dec. 2018. 626

[57] F. Guo, Y. Liu, and F. Luo, "Adaptive stabilisation of a flexible riser by 627 using the Lyapunov-based barrier backstepping technique," IET Control 628 Theory Appl., vol. 11, no. 14, pp. 2252-2260, Sep. 2017.

58 ] Z. J. Zhao, Z. J. Liu, Z. F. Li, N. Wang and J. F Yang "Control design 630 for a vibrating flexible marine riser system," J. Frankl. Inst., vol. 354, 631 no. 18 , pp. 8117-8133, 2017

59] Z. J. Zhao, X. Y. He, Z. G. Ren, and G. L. Wen, "Boundary adaptive robust control of a flexible riser system with input nonlinearities," 634 IEEE Trans. Syst., Man, Cybern., Syst., vol. 49, no. 10, pp. 1971-1980, 635 Oct. 2019.

[60] Z J. Zhao, X. Y. He, and G. L. Wen, "Boundary robust adaptive anti-saturation control of vibrating flexible riser systems," Ocean Eng., 63 vol. 179, pp. 298-306, May 2019

[61] Z. J. Zhao, S. M. Lin, D. C. Zhu, and G. L. Wen, "Vibration control 640 of a riser-vessel system subject to input backlash and extraneous dis- 64 turbances," IEEE Trans. Circuits Syst. II, Exp. Briefs, to be published. 642 doi: 10.1109/TCSII.2019.2914061.

[62] N. J. Ahmad and F. Khorrami, "Adaptive control of systems with back- 644 lash hysteresis at the input," in Proc. Amer. Control Conf., vol. 5, 1999, 645 pp. 3018-3022.

[63] W. He, T. T. Meng, D. Q. Huang, and X. F. Li, "Adaptive bound- 647 ary iterative learning control for an Euler-Bernoulli beam system with 648 input constraint," IEEE Trans. Neural Netw. Learn. Syst., vol. 29, no. 5, 649 pp. 1524-1535, May 2018

[64] Z. J. Zhao, X. Y. He, and C. K. Ahn, "Boundary disturbance 651 observer-based control of a vibrating single-link flexible manipu- 652 lator," IEEE Trans. Syst., Man, Cybern., Syst., to be published. 653 doi: 10.1109/TSMC.2019.2912900.

[65] Z. J. Liu, Z. J. Zhao, and C. K. Ahn, "Boundary constrained control 655 of flexible string systems subject to disturbances," IEEE Trans. Circuits 656 Syst. II, Exp. Briefs, to be published. doi: 10.1109/TCSII.2019.2901283. 657

[66] Z. J. Zhao, X. Y. He, Z. G. Ren, and G. L. Wen, "Output feedback 65 stabilization for an axially moving system," IEEE Trans. Syst., Man, 659 Cybern., Syst., to be published. doi: 10.1109/TSMC.2018.2882822. 660

[67] W. He and S. Zhang, "Control design for nonlinear flexible wings of 66 a robotic aircraft," IEEE Trans. Control Syst. Technol., vol. 25, no. 1, 662 pp. 351-357, Jan. 2017

68] M. M. Polycarpou and P. A. Ioannou, "A robust adaptive nonlinear control design," Automatica, vol. 32, no. 3, pp. 423-427, 1996.

[69] Z. L. Lyu, Z. Liu, K. Xie, C. L. P. Chen, and Y. Zhang, "Adaptive 666 fuzzy output-feedback control for switched nonlinear systems with sta- 667 ble and unstable unmodeled dynamics," IEEE Trans. Fuzzy Syst., to be 668 published. doi: 10.1109/TFUZZ.2019.2922165.

[70] K. X. Lu, Z. Liu, C. L. P. Chen, and Y. Zhang "Event-triggered neural 670 control of nonlinear systems with rate-dependent hysteresis input based 671 on a new filter," IEEE Trans. Neural Netw. Learn. Syst., to be published. 672 doi: $10.1109 / T N N L S .2019 .2919641$.

3

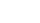


674 [71] Z Y. Fei, X, D. Wang, M. Liu, and J. Y Yu, "Reliable control for

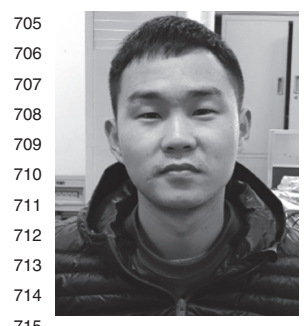

Xiuyu He (S'15) received the B.Eng. degree in automation from the Hubei University of Technology, Wuhan, China, in 2012, and the M.E. degree from the School of Automation Engineering and Center for Robotics, University of Electronic Science and Technology of China, Chengdu, China in 2016 . He is currently pursuing the Ph.D. degree with the School of Automation and Electrical Engineering, University of Science and Technology Beijing, Beijing, China.

His current research interests include distributed 716 parameter system, marine cybernetics, and robotics.

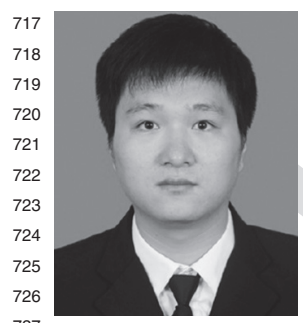

Zhijia Zhao (S'14-M'19) received the B.Eng. degree in automatic control from the North China University of Water Resources and Electric Power Zhengzhou, China, in 2010, and the M.Eng. and Ph.D. degrees in automatic control from the South China University of Technology, Guangzhou, China, in 2013 and 2017, respectively.

$\mathrm{He}$ is currently an Associate Professor with the School of Mechanical and Electrical Engineering, Guangzhou University, Guangzhou. His current 727 cybernetics, and robotics. research interests include flexible systems, marine

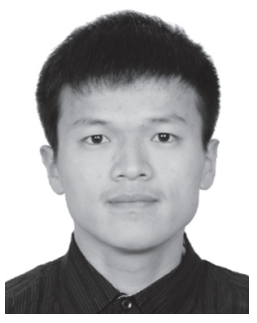

Jinya Su (S'13-M'16) received the B.Sc. degree 729 in mathematics from Shandong University, 730 Jinan, China, in 2011, and the Ph.D. degree 731 AQ6 from the Department of Aeronautical and 732 Automotive Engineering, Loughborough University, 733 Loughborough, U.K., in 2016.

Since 2015, he has been a Research Associate 735 with Loughborough University. In 2019, he joined 736 the School of Computer Science and Electronic 737

Engineering, University of Essex, Colchester, 738 U.K., as a Lecturer of Computer Science and AI. 739 His current research interests include applied machine learning, control 740 engineering, and their applications to intelligent autonomous systems, such 741 as intelligent vehicle and smart farming.

Dr. Su was a recipient of the Chinese Government Award for Outstanding 743 Self-Financed Students Abroad in 2015.

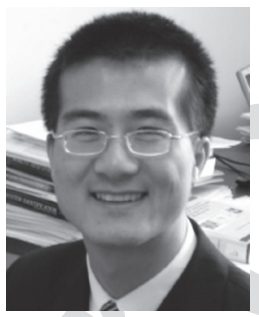

Qinmin Yang (S'05-M'10-SM'18) received the 745 bachelor's degree in electrical engineering from the 746 Civil Aviation University of China, Tianjin, China, 747 in 2001, the Master of Science degree in con- 748 trol science and engineering from the Institute of 749 Automation, Chinese Academy of Sciences, Beijing, 750 China, in 2004, and the Ph.D. degree in electri- 751 cal engineering from the University of Missouri at 752 Rolla, Rolla, MO, USA, in 2007.

From 2007 to 2008, he was a Post-Doctoral 754 Research Associate with the University of Missouri 755 at Rolla. From 2008 to 2009, he was a System Engineer with Caterpillar Inc., 756 Deerfield, IL, USA. From 2009 to 2010, he was a Post-Doctoral Research 757 Associate with the University of Connecticut, Mansfield, CT, USA. Since 758 2010, he has been with the State Key Laboratory of Industrial Control 759 Technology, College of Control Science and Engineering, Zhejiang University, 760 Hangzhou, China, where he is currently a Professor. He has also held visit- 761 ing positions in the University of Toronto, Toronto, ON, Canada, and Lehigh 762 University, Bethlehem, PA, USA. His current research interests include intel- 763 ligent control, renewable energy systems, smart grid, and industrial big data. 764

Prof. Yang has been serving as an Associate Editor for the IEEE 765 Transactions ON Systems, MAN, AND CYBERnetics-PART A: 766 Systems AND HuMANS, Transactions of the Institute of Measurement and 767 Control, and IEEE/CAA Journal of Automatica Sinica.

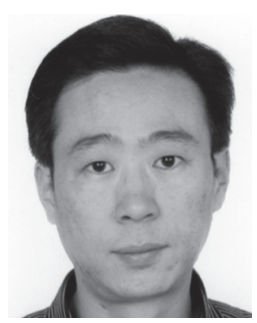

Dachang Zhu received the B.S degree in mechanics 769 design and the M.S degree in theoretical mechan- 770 ics from the Jiangxi University of Technology and 771 Science, Ganzhou, China, in 1996 and 1999, respec- 772 tively, and the Ph.D degree in mechanical engineer- 773 ing from Beijing Jiaotong University, Beijing, China, 774 in 2008 .

He was a Post-Doctoral Research Fellow with the 776 South China University of Technology, Guangzhou, 777 China, in 2012. He is currently a Full Professor 778 with the School of Mechanical and Electrical 779 Engineering, Guangzhou University, Guangzhou. His current research interests 780 include topology optimization theory and applications in compliant mecha- 781 nisms, robotics, and feedback control of dynamic systems.

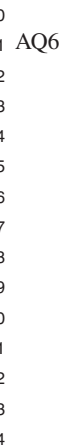

